\title{
The Complex Relationship Between Empathy, Engagement and Boredom
}

\author{
Harry J. Witchel \\ Brighton \& Sussex Medical School \\ Brighton BN1 9RY, UK \\ h.witchel@bsms.ac.uk \\ +441273873549 \\ Julian Tee \\ Brighton \& Sussex Medical School \\ J.Tee1@uni.bsms.ac.uk
}

\author{
Carlos P. Santos \\ Brighton \& Sussex Medical School \\ C.PaulinoDosSantos1@uni.b \\ sms.ac.uk \\ Nachiappan Chockalingam \\ CSHER \\ Staffordshire University, UK \\ n.chockalingam@staffs.ac.uk
}

\author{
James K. Ackah \\ Brighton \& Sussex Medical School \\ J.Ackah1@uni.bsms.ac.uk
}

\author{
Carina E.I. Westling \\ School of Media Film \& Music \\ University of Sussex, UK \\ c.e.i.westling@sussex.ac.uk
}

\begin{abstract}
In human computer interactions - especially gaming - the role of empathy has been mooted as a necessary prerequisite for higher levels of engagement and immersion. More recently other forms of engagement, including intellectual/cognitive engagement, have been proposed. In this study we present a carefully controlled dataset of human-computer interactions with a wide range of stimuli that ranged from highly engaging to boring to test these two theories. Analyzing 844 response sets to visual analogue scales (VAS) for empathy, interest, boredom, and engagement, we found that high empathy was sufficient for high engagement but is not necessary, whilst the converse was not true. We also found that empathy and boredom were incompatible with each other, but low levels of either were permissive rather than causal to the other. We conclude that there is no monotonic relationship between increasing empathy and engagement; either empathy is a sufficient (but not necessary) cause of engagement, or engagement is a necessary precursor to high empathy.
\end{abstract}

\section{CCS Concepts}

-Information systems $\sim$ Multimedia information systems - Human-centered computing HCI design and evaluation methods • Human-centered computing Laboratory experiments

\section{Keywords}

Engagement; empathy; boredom; interest.

\section{INTRODUCTION}

In the games literature, six different types of causes of

Permission to make digital or hard copies of all or part of this work for personal or classroom use is granted without fee provided that copies are not made or distributed for profit or commercial advantage and that copies bear this notice and the full citation on the first page. Copyrights for components of this work owned by others than the author(s) must be honored. Abstracting with credit is permitted. To copy otherwise, or republish, to post on servers or to redistribute to lists, requires prior specific permission and/or a fee. Request permissions from Permissions@acm.org.

ECCE '16, September 05 - 08, 2016, Nottingham, United Kingdom Copyright is held by the owner/author(s). Publication rights licensed to ACM.

ACM 978-1-4503-4244-5/16/09..\$15.00

DOI: http://dx.doi.org/10.1145/2970930.2970935 engagement have been proposed: intellectual, physical, sensory, social, narrative, and emotional [6]. This new theoretical backdrop makes engagement a family of cognitive states, rather than one monolithic state, where intellectual and emotional engagement can be separated $[11,13,14]$. In human-computer interaction (e.g. video games) empathy has been considered a necessary pre-requisite for higher levels of engagement (Figure 1, Theory 1) leading to positive experiences $[1,2]$. However, the causal direction between engagement and empathy remains controversial, as the Perceiving and Experiencing Fictional Characters (PEFiC) theory, which is used to interpret fiction and autonomous agents, suggests that engagement (and impact) can lead to empathy (Theory 3)[9]. In psychology, empathy is in general considered "the first necessary step" in engagement with helping behavior [7], but helping behavior is not included in all forms of engagement (Theory 2).

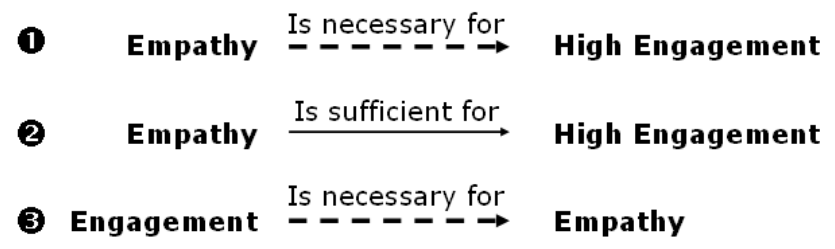

Figure 1. Three theories relating engagement to empathy.

Empathy is "the apprehension of another's inner world and a joint understanding of emotions", and it comprises i) perspective taking and ii) feeling the emotions of others [8]. In this study we test whether empathy is necessary for engagement, or merely sufficient in certain contexts, i.e. one of many potential facilitators for engagement, by using a wide range of interactive contexts.

\section{METHODS}

\subsection{Experimental volunteers and protocol}

64 adult volunteers (age $23.4 \pm 8.4$ years, mean \pm SD, 33 females) who were recruited from the university community $(70 \%$ were current students) experienced 844 3-minute long stimuli; most persons experienced 12 stimuli out of a set of 41 stimuli. After each stimulus, participants filled in VAS rating scales. All stimuli were presented in a counterbalanced order. The experimenters left the room prior to starting the stimulus, so the volunteer was alone during the presentation. 


\subsection{Stimuli and subjective rating scales}

The stimulus set included passive and interactive stimuli, with all interactions conducted using a hand-held trackball (i.e. without a keyboard). The stimulus set ranged from very interesting to extremely boring $[12,14]$ (Figure 2), including:

1) Commercial leisure games including Angry Birds and Zuma

2) Musical stimuli, including music videos, self-selected favorite music (audio-only), and unbearable music (a violin played incompetently for 3 minutes)

3) Interactive quizzes made in Flash, ranging from engaging (interesting and stimulating) to meaningless and frustrating (e.g. difficult or pointless questions where no feedback is provided after the participant answers)

4) Reading passages (in the form of reading comprehension quizzes) that ranged from interesting and engaging (best selling novels) to boring, dense and opaque to the lay reader (European Union Banking Regulations).

The four stimuli in Figures 6 and 7 are described in [13]. In brief, A5 was a photomontage of images (many were pleasant, smiling faces) that changed every six seconds, OK was a popular music video by OK Go ("This Too Shall Pass - Rube Goldberg version"), ZU was a commercial computer game called Zuma, and IPSK was a single photograph lasting 120 seconds of a ski jumper about to descend a ski jump, shot from the first person perspective (IAPS 8030).

After each stimulus, the participants filled in the Visual Analogue Scales (VAS) introduced by the phrased, "During the stimulus I just experienced", including "I felt interested", "I felt bored", "I felt empathy or emotional attachment to what I saw", and "I felt totally engaged". Each VAS ranged from 0 ("Not at all") to 100 ("Extremely"). In other fields the VAS is a reproducible scale measurement [5], and it has been defended as a reliable interval scale that has ratio property validity [4].

\subsection{Statistics and analysis}

All statistics reported here were calculated in Matlab.

\section{RESULTS AND DISCUSSION}

To understand the relationships between pairs of descriptors, bubble plots were made in which $\mathrm{X}, \mathrm{Y}$ pairs of the prevalence of the result are represented by the area of the filled circle. Figure 3 shows that engagement was very highly correlated with interest (Spearman's rho $=0.8282$ ), and that interest was both necessary and sufficient for engagement. These lay descriptors (in this data set of two-minute stimuli) are nearly substitutable.

\subsection{Empathy's relationships with boredom and engagement}

Figure 4 shows that increasing and high empathy drastically reduces the ratings of boredom. While the causal direction cannot be derived from this figure, it suggests that either empathy is sufficient to prevent boredom, or that boredom is sufficient to prevent empathy. From the activity at the lower left of the graph, it is clear that empathy is sufficient to prevent boredom, but that it is not necessary to prevent boredom, nor vice versa. The implication is that empathy and boredom are mutually exclusive (Spearman's rho $=-0.5101)$, in the sense that any empathy prevents even mid-range levels of boredom, while high boredom guarantees that no empathy is possible. Figure 5 shows the relationship between self-described empathy and engagement.

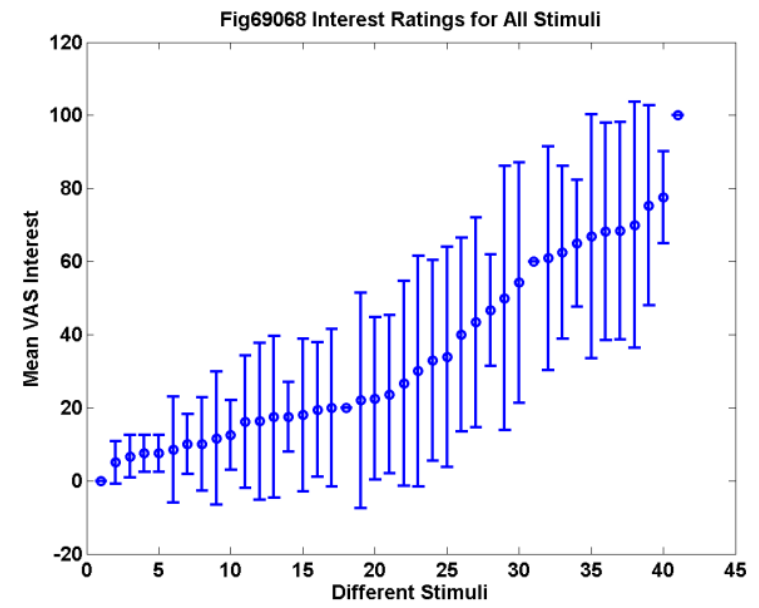

Figure 2. Mean VAS values for engagement for our collection of stimuli, ordered by mean engagement; error bars are S.D.

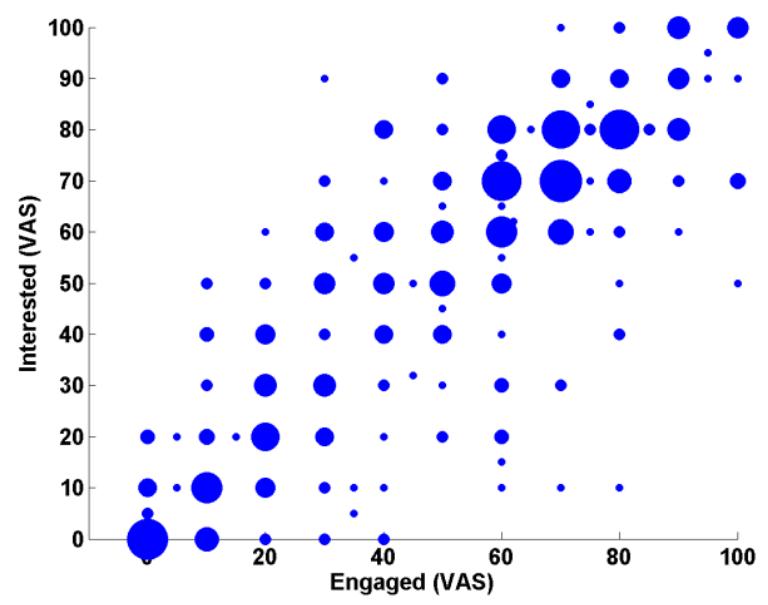

Figure 3. Relationship of subjective interest to engagement.

The lack of rating pairs in the lower right suggests that either high empathy is sufficient to imply engagement, or that low engagement is necessary to allow empathy (Spearman's rho $=$ 0.5449 ). In either event, self-described empathy is not necessary for engagement ("no boredom").

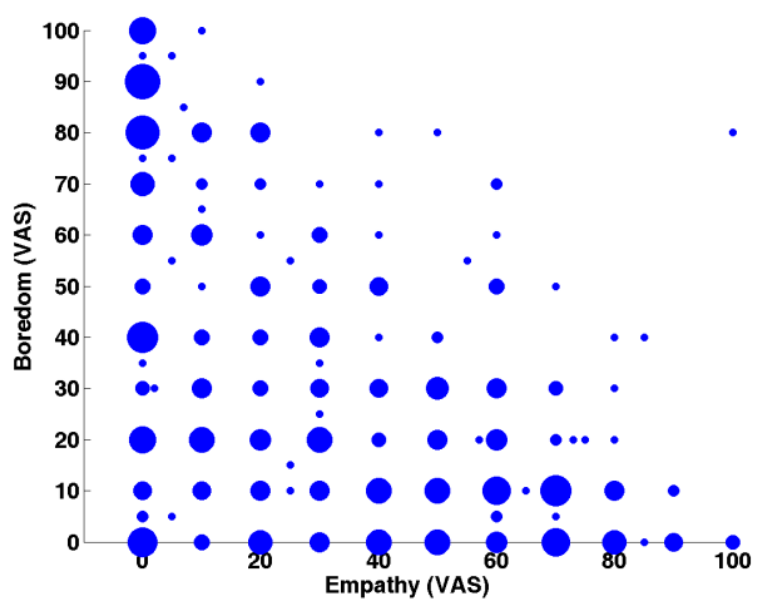

Figure 4. High empathy and high boredom are mutually exclusive, thus each is sufficient to eliminate the other. 


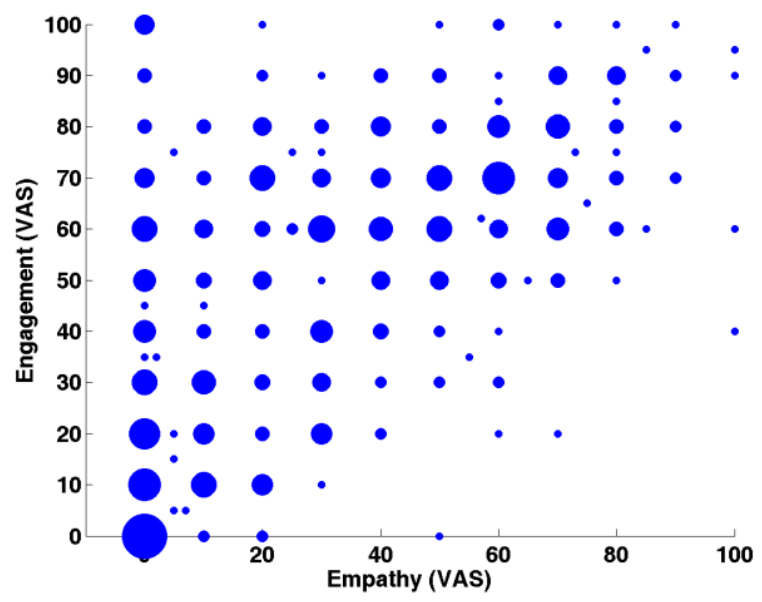

Figure 5. High empathy is sufficient for high engagement, but it is not necessary.

In order to more specifically understand how the complex relationships play out in individual circumstances, in Figures 6 and 7 we compare four stimuli and how they differentially affect empathy and engagement. The four selected stimuli differ in whether they include smiling faces, fast action, regular userinteraction, and a first person point of view. For each stimulus, the same healthy participants' ratings were compared. Kruskal Wallis tests were performed on both rating sets, and in the plot for empathy the Chi-squared $\chi^{2}(3,135)=17.5$ and $\mathrm{P}<0.001$. In the Tukey post hoc comparison (Matlab, multcompare) A5 was more empathetic than the three others, which were not statistically different from each other (Figure 6). In the engagement comparison, the Kruskal Wallis Chi-squared $\chi^{2}(3,135)=65.1$ and $\mathrm{P}<0.0001$, and the post hoc analysis showed that all were statistically different from one another except OK and ZU (Figure 7).

These results support the idea that smiling faces increase subjective empathy more strongly than a first person point of view, or user interaction. By contrast, all three of these features may contribute to subjective engagement (Figure 7). However, seemingly the most important stimulus feature for user engagement was fast action, which increased engagement synergistically when combined with user-interaction.

\section{CONCLUSIONS}

In this study we investigated the relationship between the lay use of the word empathy and the lay use of engagement and boredom by using the visual analogue scale. With the caveat that the lay use of these words is not identical to the various researcher-led uses of these word, the range of our stimuli allowed us to investigate how lay users would relate these terms under varied interaction activities. We found that, for lay usage of these words, empathy is not a pre-requisite for higher levels of engagement, counter to popular design theories about immersion [1,2]. Either user-described empathy is a sufficient but not necessary cause for engagement, or user-described engagement is a necessary cause for empathy.

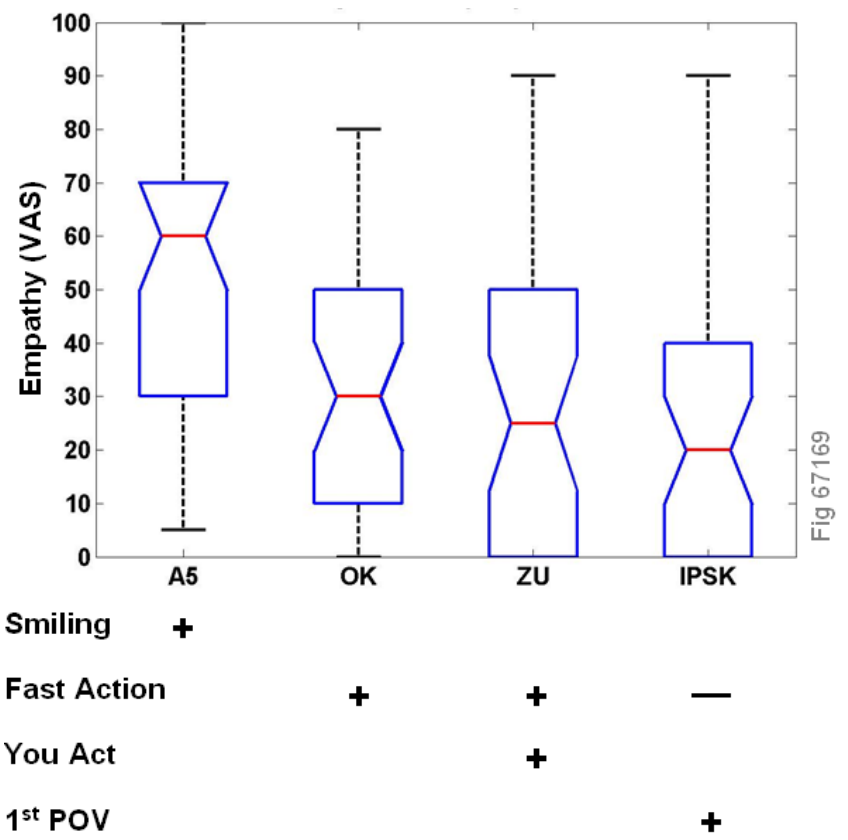

Figure 6. Comparison of empathy ratings from four specific stimuli (above), and attributes of those stimuli below. Smiling

$=$ smiling faces appear often. Fast Action $=$ image changes or events occur rapidly. Minus $=$ no activity. You Act $=$ user interaction (trackball) determines the course of events. 1st POV $=$ seen from a first person point of view. $N=30$ for each.

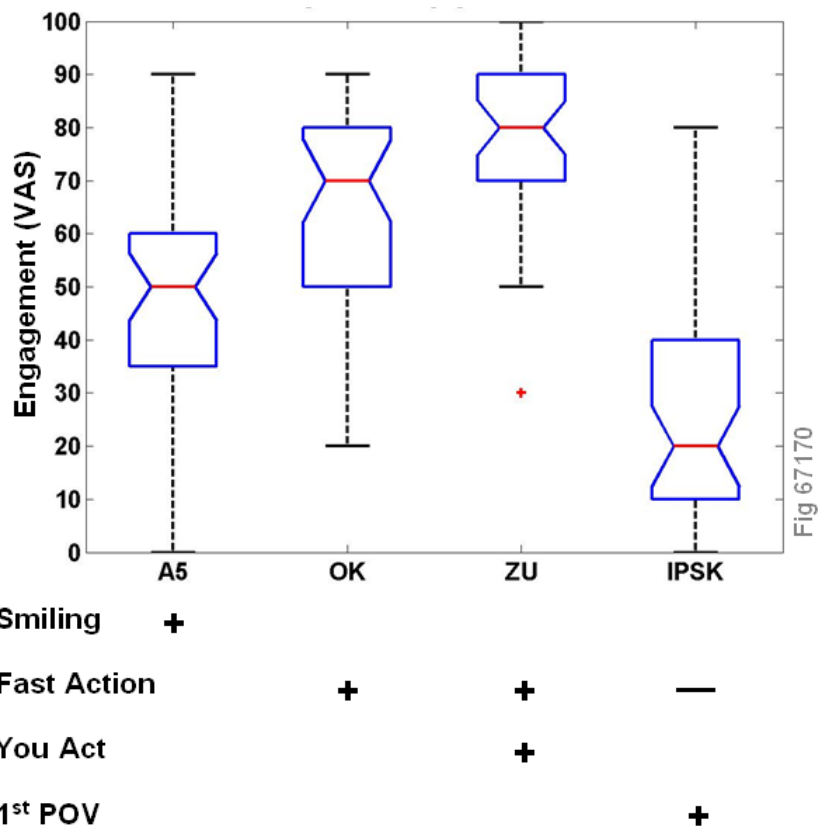

Figure 7. Comparison of engagement ratings from the same four stimuli (above), and attributes of those stimuli.

Given our experimental approach, we cannot determine the direction of causality. From Figure 1, we have provided strong evidence to support Theories 2 and 3 , and our data eliminates Theory 1 . We also found that boredom and empathy are mutually exclusive, which supports the same Theories. 
These results suggest that the current emphasis on empathy for the purpose of engendering engagement in interface and interaction design should be re-examined. A lack of empathy is not a barrier to high engagement, although such a lack may prevent immersion in gaming contexts. This fits with the cautionary tale that sometimes design attempts to foster empathy can backfire instead of raising engagement - for example, Microsoft's Office Assistant "Clippy". In brief, empathy can be used to increase engagement, but it is not the only way, the best way, or a required way to do so. Other non-obligate factors for increasing engagement may include challenge, feedback, control, and variety [10], as well as novelty, aesthetics and usability [3].

\section{ACKNOWLEDGMENTS}

We gratefully acknowledge the Wellcome Trust for funding a Biomedical Vacation Scholarship for CS (Grant 105298/Z/14/Z). We acknowledge the BSMS IRP programme for financing the IRP running costs. We are grateful to Chätrin Tolga for volunteer recruitment and data entry, and to Jacob Greaves for technical assistance. Finally we are grateful to Stephen Bruner for the initial research idea on the importance of music.

\section{REFERENCES}

[1] Brown, E. \& Cairns, P. 2004. A grounded investigation of game immersion. In CHI'04 extended abstract on Human Factors in Computing Systems (pp. 1297-1300).

[2] Lee, M. \& Kim, G.J. 2014. Empathetic video clip experience through timely multimodal interaction. Journal Multimodal User Interfaces, 8(3), pp. 273-288.

[3] O'Brien, H.L. \& Toms, E.G. 2010. The development and evaluation of a survey to measure user engagement in ecommerce environments. Journal of the American Society for Information Science \& Technology, 61(1), 50-69.

[4] Price, D.D., Staud, R., Robinson, M.E., 2012. How should we use the visual analogue scale (VAS) in rehabilitation outcomes. II: Visual analogue scales as ratio scales: An alternative to the view of Kersten et al? Journal of Rehabilitation Medicine;44:800-1.

[5] Rosier, E.M., Iadarola, M.J., Coghill, R.C., 2002. Reproducibility of pain measurement and pain perception. Pain;98:205-216
[6] Schønau-Fog, H., \& Bjørner, T. (2012). "Sure, I Would Like to Continue" A Method for Mapping the Experience of Engagement in Video Games. Bulletin of Science, Technology \& Society, 32(5), 405-412.

[7] Singer, T., \& Lamm, C. (2009). The social neuroscience of empathy. Annals of the New York Academy of Sciences, 1156(1), 81-96.

[8] Tapus, A., Mataric, M.J. and Scasselati, B., 2007. Socially assistive robotics. Robotics \& Automation Magazine, IEEE, 14(1), pp.35-42.

[9] van Vugt, H. C., Hoorn, J. F., \& Konijn, E. A. (2005). Digital bonding: Interactive and affective affordances of interface characters. Autonomous Agents and Multi-Agent Systems.

[10] Webster, J., \& Ho, H. 1997. Audience engagement in multimedia presentations. ACM SIGMIS Database, 28(2), 63-77.

[11] Witchel, H. J. 2013a. Engagement: the Inputs and the Outputs. Proceedings of Inputs-Outputs: An Interdisciplinary Conference on Engagement in HCI and Performance. ACM: New York. Article 1.

[12] Witchel, H. J., Westling, C., Needham, R., Healy, A., \& Chockalingam, N. 2013b. Mean head and shoulder heights when seated: subconscious postural cycles during discrete computerised stimuli. Proceedings of the 31st European Conference on Cognitive Ergonomics.

[13] Witchel, H. J., Westling, C., Tee, J., Healy, A., Needham, R., \& Chockalingam, N. 2014. What does not happen: Quantifying embodied engagement using NIMI and selfadaptors. Participations: Journal of Audience and Reception Studies, 11(1), 304-331.

[14] Witchel, H. J., Santos, C. P., Ackah, J. K., Westling, C.E.I., Chockalingam, N, 2016. Non-Instrumental Movement Inhibition (NIMI) Differentially Suppresses Head and Thigh Movements during Screenic Engagement: Dependence on Interaction. Frontiers in Psychology 7:157.doi: 10.3389/fpsyg.2016.00157 